\title{
Managing Complexity with Design Structure Matrices - an Operational Life Integration Challenge
}

\author{
Edward J. Repetski ${ }^{1}$, Shahram Sarkani ${ }^{1}$, Thomas A. Mazzuchi ${ }^{1}$ \\ ${ }^{1}$ George Washington University
}

\begin{abstract}
This study is exploring a way to manage complexity in the operational test and evaluation of complex systems to include the integration of human crews. The approach is two-fold: Multi-Domain Matrices (MDM) are used to visualize the organization of complex systems followed by a model based on the Multi-Layer Perceptron (MLP) being applied to the integration process of these crews. This study seeks to expand the application of model-based engineering into a new domain, operational integration of complex equipment sets with human crews. The case study used to explore this model is a simplified version of equipment for vehicle mounted military gunnery. This is to determine if such a model can provide a path for expanding the benefits of systems thinking into this domain.
\end{abstract}

Keywords: Design Structure Matrices, Multi-Domain Architecture, Domain Mapping Matrices, Complexity, Operational Test and Evaluation

\section{Introduction}

The purpose of this study is to investigate if modeling tools can improve the efficacy and efficiency of the integration of human crews with complex equipment sets during the operational lifespan. This represents moving Model-Based Systems Engineering (MBSE): 1) farther into the complex integration of multiple systems plus 2) later into the lifespan of systems to include 3) modeling human actors.

The case study used to explore this topic is the integration of new crews with various vehicles, weapons and communications platforms in a resource-constrained environ. Specifically, U.S. Army crews being organized with various equipment sets and limited time as well as resource-constrained integration support tools while preparing for operational use. The question to be addressed is: Can complexity modeling tools such as Design Structure Matrices be used to integrate humans and multiple systems in the operational life more effectively?

The value of this proposed application is the potential to improve a process that has wide use while providing a tool that is easily applied and accessible. The Army has thousands of crews for which this could be applicable and the tool proposed would be available for wide use with common software.

This expands the application of such models along those three axes; to a higher complexity level, into the operational lifetime of equipment, while modeling human behavior. The literature review that follows traces the progress along those three avenues of thought to the present. Further, the literature review touches upon a machine learning concept, the 
Part I: Managing Organizations

Multilayer Perceptron (MLP). The intent is to use MLPs as a conceptual model for the human crew's integration process.

\section{Literature Review}

Mahdi and Sievers, in their special paper pointed out that the frontiers of MBSE is pushing along the frontiers relevant to this research; more complex systems, to include human actors, and later into the operational life-spans of equipment sets (Madni and Sievers, 2018). The literature trail branches out down these three tracks to support this work. The primary focus of the literature review is on the frontiers of application of Design Structure Matrices.

\subsection{Complexity Modeling}

The idea of using matrix models to understand the complex has a long history. The resulting matrices offer two benefits in application, they are easily understandable visibly and easily manipulated mathematically. Matrices are intuitive to understand and can help explain connections in systems to create common understanding among teams of people. With the finite number of connections between elements these complexity modeling matrices have an appeal for calculations because they are "nearly decomposable" (Simon, 1962). That is, many cells of the matrix are zero or close enough to simplify calculations. Further, matrices can be reordered to move the location of interactions closer along the diagonal in a matrix. This allows the tasks that are most related to be grouped to be more efficiently iterated (Steward, 1981). These Design Structure Matrices can be applied to products and organizations as well as processes. Originally, this idea is used to group the components of a new design into elements and this use has expanded into other applications to include; expansion in ways to track communications in organizations, or to analyze organization of tasks or processes (Eppinger and Browning, 2012). These ideas can be expanded into the interactions between component elements in the complex by looking at the resulting, if not square, intersecting matrices between DSMs to analyze multi-domain architectures. These Domain Mapping Matrices help to understand the connections between domains (Morelli et al., 1995). These Multi-Domain Architecture applications have shown promise to help create common understandings of complex systems and the ideas of summing and taking differences between DSMs to show connections between related systems to understand commonality and difference allow us to build companion matrices for each integrating tool (Eppinger and Browning, 2012). The potential to use that common understanding and simplicity of manipulation to better understand and manage and improve complex systems has shown great potential as the application of DSM (Browning, 2016).

\subsection{Later Life-Cycle}

Most applications of MBSE have focused on the design and production stages of products. Some efforts have been made int the verification and validation process ( $\mathrm{Li}$ et al., 2019). Also, the application of MBSE to Operational Test Planning show the benefits of models to improve later life-cycle process in domains where expert opinion has held sway for want of a means to apply rigor (Bjorkman et al., 2013a) (Bjorkman et al., 2013b). In fact the enhancement or validation of expert opinion is highly related to this work. Further the idea 


\section{E. J. Repetski, S. Sarkani, T. A. Mazzuchi}

of using digital "twin" models during the operational lifecycle is becoming a reality (Madni et al., 2019). Models for application in the operational life-span are expanding.

\subsection{Modeling humans.}

Humans have been included in DSMs for various purposes but the applications have been focused on communication links and process flow vice behavior. Process DSMs can look at how humans can be included but the focus is on linkage vice behavior (Eppinger and Browning, 2012). None of the exemplar models attempted to model human behavior so much as the linkages between them or them and the work.

\subsection{Multi-Layer Perceptron.}

The Multi-Layer Perceptron (MLP)is a tool in Machine Learning. The MLP is a universal approximator. To understand how a perceptron learns is to examine how layers behave when they are exposed to data, check their weights through a backward analysis to refine these weights and repeat. Each MLP learning cycle is called an epoch (Alpaydin, 2014). The analogy of a crew as an MLP allows us to evaluate how each crew learns by iterative exposure. Each Integration Training Event Becomes an epoch, optimal integration being the objective of this learning. Each integration tool has different impacts on various parts of the model so the model must sum iterations by tool type. If we assume a learning rate by iteration for each integration tool and also include a momentum term that shows how the impact of each epoch or iteration changes with repetition, we can model the integration process.

\section{Case Study}

The case study against which this model is built and will be tested is the integration of human crews with rapidly assembled equipment sets for use. The model provided here is simplified and generic using terms like vehicle and weapon not specific vehicles and weapons for ease of clarity and broader application. The intent here is to show how the model is to be built, refined, analyzed and applied.

Each year the Army trains vehicle mounted crews for operational deployment. Prior to deployment the crews typically meet and are married to their equipment set a matter of months before operational deployments. They have access to a variety of integration tools ranging from the simplest, hands on work with the equipment in isolation to the application of various simulations and up to the most complex practice events involving live-fire ranges. These integration tools come with great variance in cost and impact.

\subsection{Crews}

In this case study, the crews each have three Soldiers; the Leader, the Gunner and the Driver, a very common configuration. The Leader (or Vehicle Commander) is most senior and responsible for all actions of the crew. Training the junior Soldiers and integrating them into a team is the Leader's responsibility. These can range from junior NonCommissioned Officers to Senior Officers with widely varying experience in crew operations. The Leader gives direction to the crew and is responsible for the 


\section{Part I: Managing Organizations}

communications kit, here me model this with FM Radio, a Digital Communications Platform, and an Intercom System.

The Gunner is normally the second in seniority and controls the crew in the leader's absence or incapacitation. The Gunner can range from junior enlisted to junior NonCommissioned Officer and is primarily responsible for the large weapon typically mounted on top of the vehicle.

The Driver is the junior member of the team, almost always a junior enlisted Soldier. The Driver is primarily responsible for the vehicle to include both operation and maintenance.

\subsection{Equipment.}

The equipment set used here is deliberately generic and represent typical elements vice naming a variety of components. The case study uses the most common arrangement, unstabilized gunnery. These have the fewest elements and also typically lack uniform training approaches. This is useful as these crews have more varied use of integration tools which will enable a richer data set. The equipment set includes a vehicle, a weapon, a radio, a digital communications platform, an intercom system and night vision capability.

- Vehicles include trucks of multiple sizes and types.

- The weapon is normally a medium or large caliber machine gun or automatic grenade launcher.

- The term radio can represent any of a number of radio configurations in common use, predominantly FM Radios.

- The Digital Communications System provides means for exchange of complex data between vehicles and typically include an integrated GPS that can display friendly locations, control measures defining where Soldiers should and should not be and perhaps location of hostile forces. This also provides a texting and reporting system.

- An intercom system of some sort is integrated into the vehicle to link the crew during operations.

- Lastly, the kit includes night vision capability. An integral part of Army tactics is the use of various night vision equipment both weapon and Soldier mounted.

A generic equipment model here provides a great example of the complexity while being understood quickly in the visual model.

\subsection{Integration Tools.}

Various integration tools are available to support crew integration. These vary from the simplest with low to no cost to expensive live-fire events. Crews typically progress through a series of events that build toward a live-fire qualification, the Operational Test.

Units assemble the equipment set and crew a few months before the Operational Test with vehicle and Soldiers arriving to build the unit. The crew starts with a weekly process of working with the equipment in a stand-alone mode, that is driving the vehicle in isolation or sitting in the vehicle practicing the steps. This has the lowest cost.

Simulation-based training. The Army uses simulations to train crews. For unstabilized gunnery the use of simulations is optional and the application is uneven. Simulation-based 


\section{E. J. Repetski, S. Sarkani, T. A. Mazzuchi}

training has some cost and the systems available are limited so access may only be available for discrete periods of time.

Live-Fire training is widely assumed to be the best integration tool but the higher cost of range operation, vehicle use and ammunition consumption means that this integration event is only funded for each crew once. These events follow a series of tables that are laid out in a manual and are normally done the day and night prior to the operational test.

\subsection{Operational Test.}

The operational test for a crew is Gunnery Qualification. The objective is to pass a standard test designed to ensure that all crews are minimally lethal. Further the test has a maximum score. Crews scoring a maximum are the best integrated.

\section{Problem Statement}

Maximizing the efficacy of crews for operational use is constrained by time and the cost of integration tools like ranges, ammunition, simulation support, etc. This study examines if Decision Support Matrices, specifically Multi-Domain Matrices can improve the application of integration resources to maximize the integration of a human crews with various equipment packages to improve preparation for operational use.

\section{Methodology}

The Methodology first creates a Multi-Domain Matrix (MDM) of the system and integration tools then Creates a Multi-Layer Perceptron (MLP) model of crew integration with each iteration of an integration tool (training event) as an epoch. Finally, the impact of all integration events is Summarized and subtracted from the integration requirement in the base model.

\subsection{Multi-Domain Matrix of the System Model}

This study applies DSM to the case study. A simplified model is shown here (Figure 1.) with a component structure as detailed earlier. The model uses an input on rows (IC) convention. The values of the cells are; zero meaning no integration requirement, 1 as minimal integration, 2 as significant integration and 3 as essential integration. The values here are examples for building the model. While common sense was generally applied these values are not critical to understanding the methodology and will be refined using data during later stages of this investigation. 
Part I: Managing Organizations

\begin{tabular}{|r|c|c|c|c|c|c|c|c|c|c|}
\hline & 1 & 2 & 3 & 4 & 5 & 6 & 7 & 8 & 9 \\
\hline Gumner & 1 & $\mathrm{X}$ & 3 & 2 & 1 & 3 & 1 & 1 & 2 & 3 \\
\hline Leader & 2 & 3 & $\mathrm{X}$ & 3 & 2 & 2 & 3 & 3 & 2 & 2 \\
\hline Driver & 3 & 2 & 3 & $\mathrm{X}$ & 3 & 1 & 2 & 2 & 2 & 2 \\
\hline Vehicle & 4 & & & & $\mathrm{X}$ & 1 & & & & \\
\hline Weapon & 5 & & & & 3 & $\mathrm{X}$ & & & & 2 \\
\hline Radio & 6 & & & & 1 & 3 & $\mathrm{X}$ & 3 & 3 & \\
\hline Lower Tactical Internet & 7 & & & & 1 & & 3 & $\mathrm{X}$ & & \\
\hline Intercom & 8 & & & & 1 & & 3 & & $\mathrm{X}$ & \\
\hline Night Vision System & 9 & & & & & 1 & & & & $\mathrm{X}$ \\
\hline
\end{tabular}

Figure 1. Base Model (Input in Rows)

This creates a simple Multi-Domain Architecture as shown in Figure 2. Here the three areas are detailed; the DSMs for the Crew $(3 \times 3)$ and the Equipment $(6 \times 6)$ and the Integration between the Crew and Equipment as a DMM $(3 \times 6)$. For the Integration of the Equipment, the connections are important but are mechanical or electrical and are made following defined processes. The interest here is the DSM of the Crew and the DMM; the $3 \times 9$ Matrix in Figure 3. This matrix can be seen as the integration requirement and will be called the Combined Integration Matrix (CIM).

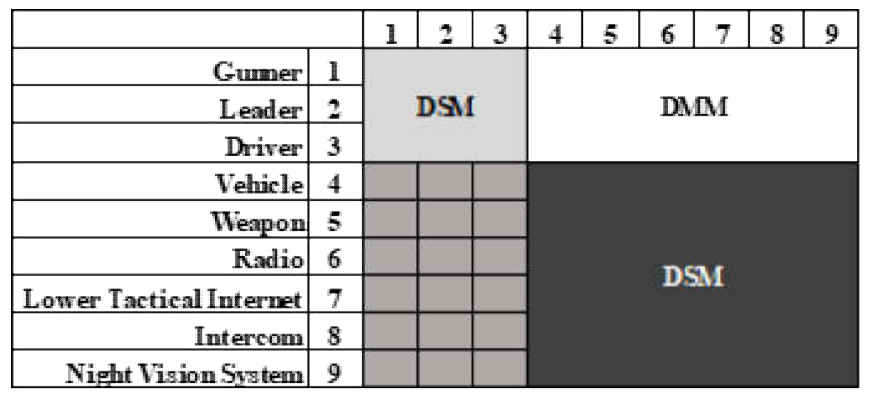

Figure 2. DSMs and DMM of the Multi-Domain Architecture

\begin{tabular}{|r|c|c|c|c|c|c|c|c|c|c|}
\hline & 1 & 2 & 3 & 4 & 5 & 6 & 7 & 8 & 9 \\
\hline G unner & 1 & $\mathrm{X}$ & 3 & 2 & 1 & 3 & 1 & 1 & 2 & 3 \\
\hline Leader & 2 & 3 & $\mathrm{X}$ & 3 & 2 & 2 & 3 & 3 & 2 & 2 \\
\hline Driver & 3 & 2 & 3 & $\mathrm{X}$ & 3 & 1 & 2 & 2 & 2 & 2 \\
\hline
\end{tabular}

Figure 3. The $3 \times 9$ Combined Integration Matrix (CIM).

\subsection{Crew Integration as an MLP}

For each integration tool a related $(3 \times 9)$ matrix is constructed with credit for which integration requirement are addressed. Such a model is shown in Figure 4. The example is a simple simulation trainer that only addresses the Leader, the Gunner, the Weapon and the Intercom. Note that nodes where integration is impacted have ones while those without impact have a quantity of zero. 


\section{E. J. Repetski, S. Sarkani, T. A. Mazzuchi}

\begin{tabular}{|r|c|c|c|c|c|c|c|c|c|c|}
\hline & 1 & 2 & 3 & 4 & 5 & 6 & 7 & 8 & 9 \\
\hline Gunner & 1 & 0 & 1 & 0 & 0 & 1 & 0 & 0 & 1 & 0 \\
\hline Leader & 2 & 1 & 0 & 0 & 0 & 1 & 0 & 0 & 1 & 0 \\
\hline Driver & 3 & 0 & 0 & 0 & 0 & 0 & 0 & 0 & 0 & 0 \\
\hline
\end{tabular}

Figure 4. The $3 \times 9$ Integration Tool Matrix (ITM)

Integration of the crew is shown by a different Integration Tool Matrix (ITM) (like Figure 4.) for each integration tool. An advantage is how this model shows the impacts of each integration tool by a simple 1 or 0 convention.

\subsection{Summation and Differences of Matrix Models.}

Next, the model treats the Combined Integration Matrix as the Requirement and the summation of all the Integration Tool Matrices as meeting those requirements. A perfectly integrated crew then has an integration matrix with all zeros meaning integration is complete.

In greater detail, the Integration Tools Matrices (ITM) are summed with a multiplier and a growing factor to show the reducing impact of each integration tool over time and epochs introducing the idea of Momentum. The integration of each Tool (A,B,C,D) where the ITM is defined as $(\mathbf{A}, \mathbf{B}, \mathbf{C}, \mathbf{D})$ and the total integration factor of each tool as a matrix is depicted as (AT,BT,CT,DT) that is the matrix value once it is summarized over all iterations (epochs). AT is shown by the summation of a learning rate (LR) times the summed value of the matrix divided by the iteration number. Each ITM has a different, associated Learning Rate designated with a subscript. The increasing denominator inside the summation introduces the concept of momentum, the theory that a tool is most impactful at first use and its value decreases with subsequent use. Equation (1) shows this Value.

Total Integration of $\mathrm{A}=\mathbf{A} \mathbf{T}=L R_{A} \sum_{\mathbf{1}}^{n}\left(\frac{\mathbf{1}}{i}\right) \boldsymbol{A}$ (1)

Next the Summation of all the Total Integration Matrices is subtracted from the Combined Integration Matrix (requirement matrix) and set equal to zero. This is shown in Equation (2). In both equations bold letters represent matrices.

$\boldsymbol{C I M}-\left(L R_{A} \sum_{1}^{n}\left(\frac{1}{i}\right) \boldsymbol{A}+L R_{B} \sum_{1}^{p}\left(\frac{1}{j}\right) \boldsymbol{B}+L R_{C} \sum_{1}^{q}\left(\frac{1}{k}\right) \boldsymbol{C}+L R_{D} \sum_{1}^{r}\left(\frac{1}{l}\right) \boldsymbol{D}=\mathbf{0}(2)\right.$

Lastly, the process must be optimized for cost and time. The time and dollar cost of each iteration of each tool will constrain the model to optimal integration at the lowest costs.

\section{Way Forward}

With a tentative model in place, the introduction of the first data set will allow that model to be refined. Various elements require refinement. First an initial value for the learning rates can be determined from the data.

Secondly, the model of the momentum factor (or diminishing return of value of iterations) is an assumption that need be investigated with data. Perhaps learning increases from iteration 1 to 2 then declines by some function. The data will empower a refinement of that element. The use of the factor of one divided by the iteration number is a starting point but the shape of that curve will have to be refined. 


\section{Part I: Managing Organizations}

Once a combined model with updated learning rates and a refined momentum process is added, the entire model needs to be tested against validation data to determine the extent that the expected patterns exist and if the information gleaned improves understanding of the process.

Once a functioning integration model is in place the effort to seek optimal use of resources can be introduced in terms of time and dollars.

The ultimate objective is two-fold. First is to deliver a spreadsheet-based tool that can be applied to unique situations to better inform the use of all training resources. Secondly, to demonstrate how systems thinking and model-based approaches can have broader application.

\section{References}

Alpaydin, E., 2014. Introduction to Machine Learning, 3rd ed. MIT Press, Boston.

Bjorkman, E.A., Sarkani, S., Mazzuchi, T.A., 2013a. Using model-based systems engineering as a framework for improving test and evaluation activities. Syst. Eng. 16, 346-362.

Bjorkman, E.A., Sarkani, S., Mazzuchi, T.A., 2013b. Test and evaluation resource allocation using uncertainty reduction. IEEE Trans. Eng. Manag. 60, 541-551.

Browning, T.R., 2016. Design Structure Matrix Extensions and Innovations: A Survey and New Opportunities. IEEE Trans. Eng. Manag. 63, 27-52.

Eppinger, S.D., Browning, T.R., 2012. Design Structure Matrix Methods and Applications, 1st ed. MIT Press, Boston.

Li, L., Soskin, N.L., Jbara, A., Karpel, M., Dori, D., 2019. Model-Based Systems Engineering for Aircraft Design With Dynamic Landing Constraints Using Object-Process Methodology. IEEE Access 7, 61494-61511.

Madni, A., Madni, C., Lucero, S., 2019. Leveraging Digital Twin Technology in Model-Based Systems Engineering. Systems 7, 7.

Madni, A.M., Sievers, M., 2018. Model-based systems engineering: Motivation, current status, and research opportunities. Syst. Eng. 21, 172-190.

Morelli, M.D., Eppinger, S.D., Gulati, R.K., 1995. Predicting Technical Communication in Product Development Organizations. IEEE Trans. Eng. Manag. 42, 215-222.

Simon, H.A., 1962. The Architecture of Complexity, in: Proceedings of the American Philosophical Society. pp. 467-482.

Steward, D. V, 1981. The Design Structure System: A Method for Managing the Design of Complex Systems, IEEE TRANSACTIONS ON ENGINEERING MANAGEMENT.

Contact: Edward J. Repetski, George Washington University, School of Engineering and Applied Science, 1 Old Oyster Point Road, Suite 220, Newport News, VA, 23602. Phone: 833-330-1454, Fax: 888-969-4851, edward repetski@gwu.edu 